\title{
Recent results on soft QCD topics, and jet, V+jet and photon production from ATLAS
}

\author{
Ewa Stanecka ${ }^{1,2, a}$ \\ ${ }^{1}$ Institute of Nuclear Physics Polish Academy of Sciences \\ ${ }^{2}$ on behalf of the ATLAS Collaboration
}

\begin{abstract}
This paper presents selection of recent QCD related measurements from the ATLAS Experiment at the Large Hadron Collider at CERN. The results on the charged particle production, underlying event properties, prompt photon production, charged particle multiplicity inside jets and $\mathrm{Z}$ boson production in association with up to four jets are presented. The measurements allowed us to cross-check several Monte Carlo generators with data at centre-of-mass energies of 8 and $13 \mathrm{TeV}$.
\end{abstract}

\section{Introduction}

The ATLAS experiment [1] performs studies of proton-proton ( $p p$ ) collisions at the Large Hadron Collider (LHC) [2] at CERN. The LHC collides bunches of protons at the unprecedented centre of mass energy $(\sqrt{s})$ of 7,8 and $13 \mathrm{TeV}$. The peak instantaneous luminosity recorded by the time of the conference was about $12 \times 10^{33} \mathrm{~cm}^{-1} \mathrm{~s}^{-2}$ [3].

The ATLAS detector is composed of several sub-detectors organised in layers. The closest to the proton beams collision point is the high precision tracking system, next come calorimeters and then muon chambers. The ATLAS experiment is a general purpose detector designed to investigate a wide range of particle physics, which can be generally divided into searches for new phenomena and the precision measurements. The precision measurements are very important as they improve our understanding of the Standard Model (SM) of particle physics, give better knowledge of background processes for new physics signals and also provide an important benchmark for the detector performance. For these reasons the precision measurements are repeated at each newly accessible $p p$ collision energy. In this report a selection of recently published ATLAS SM measurements related to the production of primary charged particles, hadronic jets, photons, and jets associated with a $\mathrm{Z}$ boson is reviewed.

\section{Measurements of the underlying-event at $13 \mathrm{TeV}$.}

The underlying event (UE) is the activity accompanying any hard scattering in a collision event, including multiple parton interactions, final state radiation, initial state radiation as well as the partons not participating in hard scattering process. The UE is an irreducible background at the LHC as it is not possible to separate UE processes from the hard process on an event by event basis.

ae-mail: ewa.stanecka@ifj.edu.pl 
The underlying event activity was measured by the ATLAS experiment with $13 \mathrm{TeV}$ data with a leading particle method [4]. The leading particle is defined as a charged particle with the highest $\mathrm{p}_{\mathrm{T}}$. The direction of the leading particle is used to define regions in the azimuthal plane that have different sensitivity to the UE. The azimuthal angular difference $(\triangle \phi)$ between charged particles and the leading particle is used to define three azimuthal regions: toward $\left(|\Delta \phi|<60^{\circ}\right)$, transverse $\left(60^{\circ}<|\Delta \phi|<120^{\circ}\right)$ and away regions $\left(|\Delta \phi|>120^{\circ}\right)$. The transverse region is sensitive to the UE activity since it is perpendicular to the direction of the hard scatter and hence it is expected to have less activity from the hard scattering process compared to the away region.

A measurement of charged-particle distributions sensitive to the properties of the underlying event was performed on the data sample corresponding to an integrated luminosity of $170 \mu \mathrm{b}^{-1}$. The data was collected during a special LHC fill with a low expected mean number of interactions per bunch crossing, same as for the analysis described in Section 3. The event and track selections used in the analysis follow those described in [5], with the only additional requirement which is the presence of the leading particle with $\mathrm{p}_{\mathrm{T}}$ of at least $1 \mathrm{GeV}$. The measured distributions are compared with predictions from Monte Carlo event generators. In Figure 1 the average scalar sum of charged-particle transverse momenta, $\Sigma p_{T}$, for transverse and toward regions is presented as a function of the leading particle $\mathrm{p}_{\mathrm{T}}$. In the transverse region sum $\mathrm{p}_{\mathrm{T}}$ shows a gradual increase, rising to an approximately constant plateau for $\mathrm{p}_{\mathrm{T}}$ of the leading particle greater than $6 \mathrm{GeV}$. For higher values of the leading particle $\mathrm{p}_{\mathrm{T}}$, the toward region includes contributions from the jet-like activity, which results in a gradual rise of the average sum $\mathrm{p}_{\mathrm{T}}$ density [4].
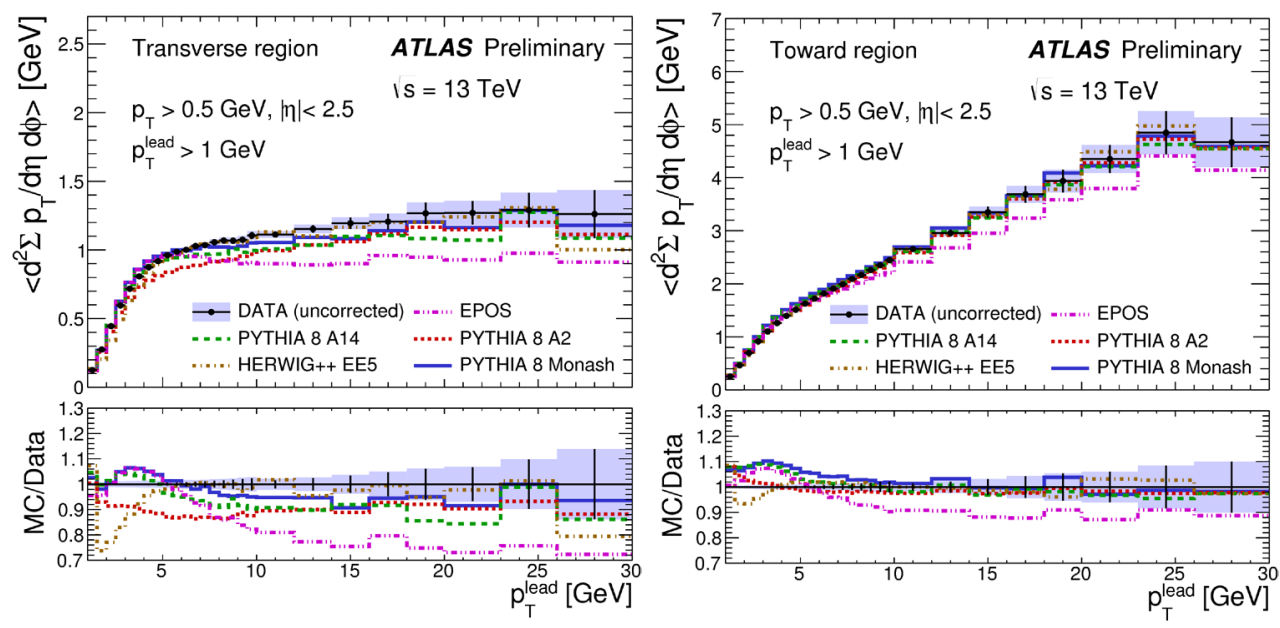

Figure 1: Comparison of detector level data and MC predictions for average charged-particle multiplicity density values (left column) and average scalar $\mathrm{p}_{\mathrm{T}}$ sum density of tracks (right column) as a function of leading particle transverse momentum, $\mathrm{p}_{\mathrm{T}}^{\text {lead }}$, in the transverse (left) and toward (right) regions. The bottom panels in each plot show the ratio of MC predictions to data. The shaded bands represent the combined statistical and systematic uncertainties, while the error bars show the statistical uncertainties [4].

Several Monte Carlo (MC) predictions were compared with the data. Pythia 8 A14, Monash and HeRwIG++ UEEE5 models are closest to data in the plateau part of the transverse region, but none of them describe the initial part well. The Epos generator predicts significantly less activity at 
higher transverse momenta of the leading particle, which is a consequence of the absence of semi-hard minimum bias processes in the model. In general, none of the models is very discrepant from the data, building a confidence in the multiple parton interactions (MPI) description used in those generators [4].

\section{Measurement of charged-particle spectra at $13 \mathrm{TeV}$}

Soft interactions are characterised by low transverse momentum transfer and cannot be calculated by perturbative Quantum Chromodynamics (QCD). The predictions for low momentum transfer processes are made with phenomenological models inspired by QCD, implemented in MC event generators. The observables connected to the production of charged-particles provide experimental probes of strong interactions at low momentum transfers and good benchmark for phenomenological models.

Measurement of the charged particles production in proton-proton collisions at $\sqrt{s}=13 \mathrm{TeV}$ was performed by the ATLAS experiment [6]. The data used for the analysis correspond to an integrated luminosity of $151 \mu \mathrm{b}^{-1}$. The data were recorded during special runs with low beam currents and reduced focusing to give a mean number of interactions per bunch crossing of 0.005 . Particles selected for the analysis are required to have a transverse momentum $\left(\mathrm{p}_{\mathrm{T}}\right)$ greater than $100 \mathrm{MeV}$ and an absolute pseudorapidity $(|\eta|)$ less than 2.5 . Events containing at least two charged particles satisfying the above kinematic criteria were used to measure the charged-particle multiplicity $\left(\mathrm{N}_{\mathrm{ch}}\right)$, its dependence on transverse momentum and pseudorapidity, and the dependence of the mean transverse momentum on multiplicity. Figure 2 presents these measurements (black dots) corrected for detector effects and compared to the predictions from several MC event generators (coloured curves). The vertical bars represent the statistical uncertainties, while the shaded areas show statistical and systematic uncertainties added in quadrature. The lower panel in each figure shows the ratio of the MC simulation to data. The MC generators and their tunes used in the analysis are listed in Table 1.

The measured mean number of charged particles per unit pseudorapidity in the region $|\eta|<0.2$ is $6.422 \pm 0.096$ with a negligible statistical uncertainty. Significant differences are observed between the measured distributions and tested MC predictions. Amongst the models considered, Epos has the best overall description of the data. Pythia 8 A2 and Pythia 8 Monash provide a reasonable overall description, whereas QGSJET-II does not describe $<p_{T}>$ vs. $\mathrm{N}_{c h}$ well, but provides a reasonable level of agreement for other distributions [6].

The presented measurement was combined with previous results to shed light on the evolution of charged-particle multiplicities with centre-of-mass energy, which so far is poorly constrained. The mean number of primary charged-particles after the correction for the contribution from strange baryons $(6.500 \pm 0.099)$ is compared to previous measurements at different centre-of-mass energies in Figure 3.

Table 1: Summary of MC generators used for comparison with the data. The generator, its version, the corresponding tune and the parton distribution function are given [6].

\begin{tabular}{llll}
\hline Generator & Version & Tune & PDF \\
Pythia 8 & 8.185 & A2 & MSTW2008LO \\
Pythia 8 & 8.186 & Monash & NNPDF2.3LO \\
Epos & LHCv3400 & LHC & - \\
QGSJET-II & II-04 & default & -
\end{tabular}




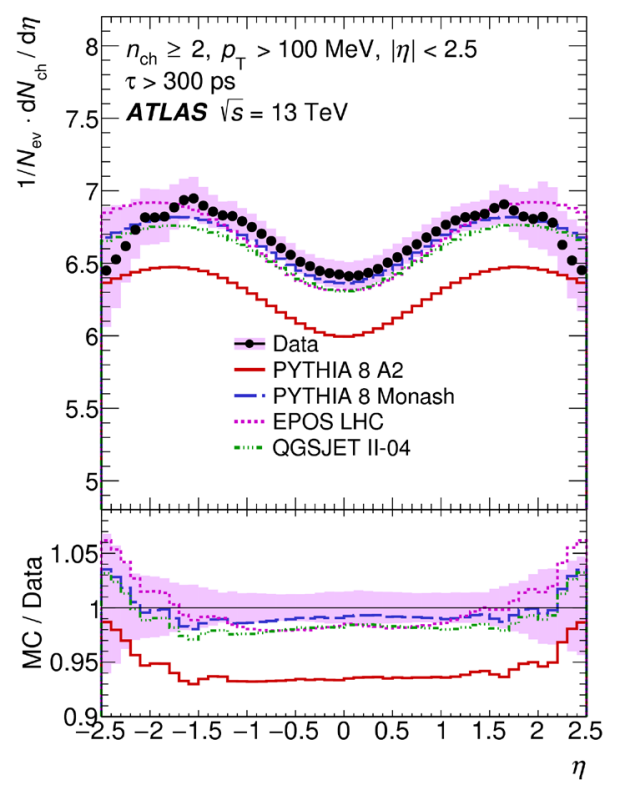

(a)

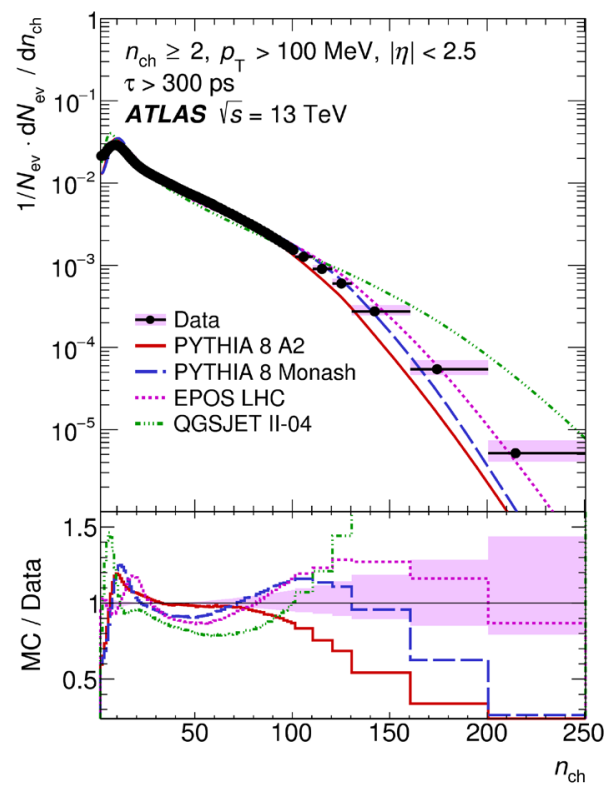

(c)

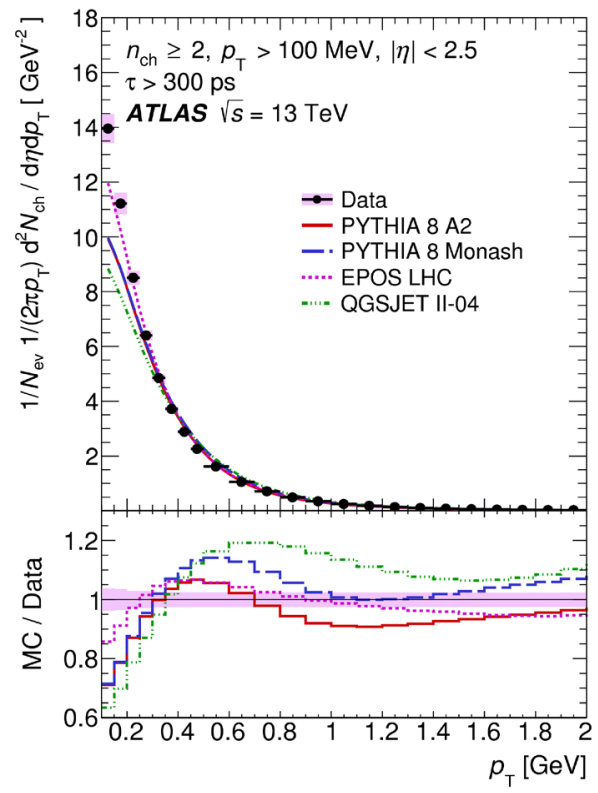

(b)

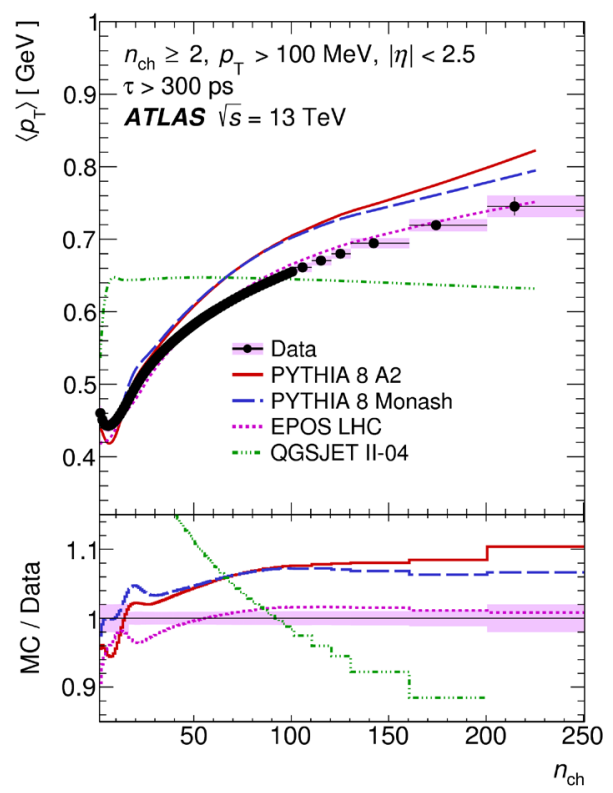

(d)

Figure 2: Primary charged-particle multiplicities as a function of (a) pseudorapidity $\eta$ and (b) transverse momentum $\mathrm{p}_{\mathrm{T}}$. The distribution of the primary charged-particle multiplicity $\mathrm{n}_{c h}(\mathrm{c})$ and the mean transverse momentum $<p_{T}>$ versus $\mathrm{n}_{c h}$ (d) for events with at least two primary charged-particles with $\mathrm{p}_{\mathrm{T}}>100 \mathrm{MeV}$ and $|\eta|<2.5[6]$. 


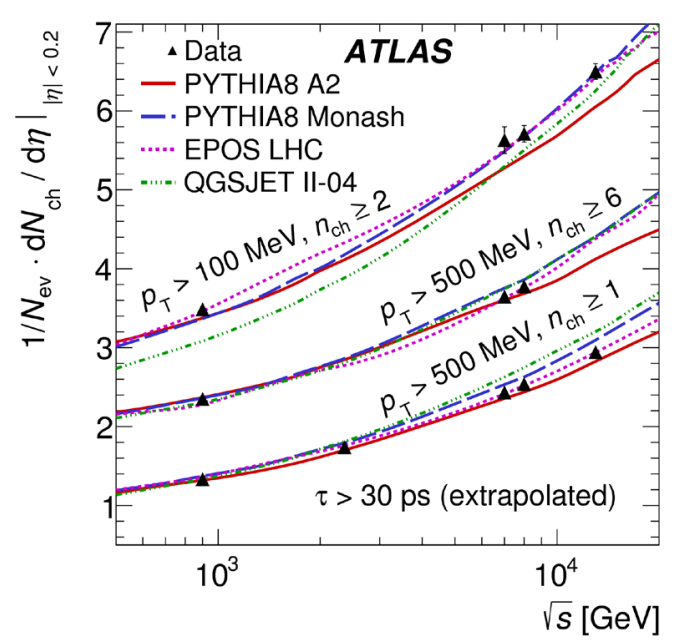

Figure 3. The average primary charged-particle multiplicity in $p p$ interactions per unit of pseudorapidity $\eta$ for $|\eta|<0.2$ as a function of the centre-of-mass energy $\sqrt{s}$. The values for the other pp centre-of-mass energies are taken from previous ATLAS analyses. The data are shown as black triangles with vertical errors bars representing the total uncertainty. They are compared to various MC predictions which are shown as coloured lines [6].

\section{Charged-particle multiplicity inside jets at $8 \mathrm{TeV}$}

The number of charged particles inside jets is often used as a discriminant for identifying the quark or gluon nature of the initiating parton and it is sensitive to both the perturbative and non-perturbative components of the jet formation.A measurement of the average number of charged particles with $\mathrm{p}_{\mathrm{T}}$ $>500 \mathrm{MeV}$ inside high-momentum jets was performed on a data sample of $20.3 \mathrm{fb}^{-1}$ recorded in $p p$ collisions at $\sqrt{s}=8 \mathrm{TeV}$ [7]. The events selected for analysis are required to have at least two central jets $(|\eta|<2.1)$, so that their charged particles are within the acceptance of the tracking system. The jets considered are clustered using the anti- $k_{t}$ jet algorithm with the radius parameter $\mathrm{R}=0.4$ and have transverse momenta from $50 \mathrm{GeV}$ up to and beyond $1.5 \mathrm{TeV}$. The jet with smaller absolute pseudorapidity is classified as more central, and the jet with larger $|\eta|$ is classified as a more forward jet. The more forward jet tends to be correlated with the parton with higher longitudinal momentum fraction $x$, and is less likely to be a gluon-initiated jet.

The reconstructed charged-particle multiplicity distribution is unfolded to remove distortions from detector effects and the resulting charged-particle multiplicity is compared to several jet formation models, as shown in Figure 4a. Comparisons at a particle level between the measured average chargedparticle multiplicity and various MC models show significant differences between the simulations using tunes for Run 1 (first season of the LHC data taking) and the data, but the Run 2 (current season of the LHC data taking) tunes for both PyтHIA 8 and HeRwig++ significantly improve the modelling of the average $\mathrm{n}_{\text {charged }}$.

The analysis shows the difference in the average charged-particle multiplicity between the more forward and the more central jets. This effect is sensitive to the difference between the number of quark and gluon constituents. The average difference, combined with the gluon fraction, was used to extract the average charged-particle multiplicity for quark- and gluon-initiated jets separately [7]. The charged-particle multiplicity in the extracted gluon-initiated jets is higher than the corresponding quantity for the quark-initiated jets and the MC generators accurately model the jet $\mathrm{p}_{\mathrm{T}}$ dependence observed in the data, see Figure $4 b$. 


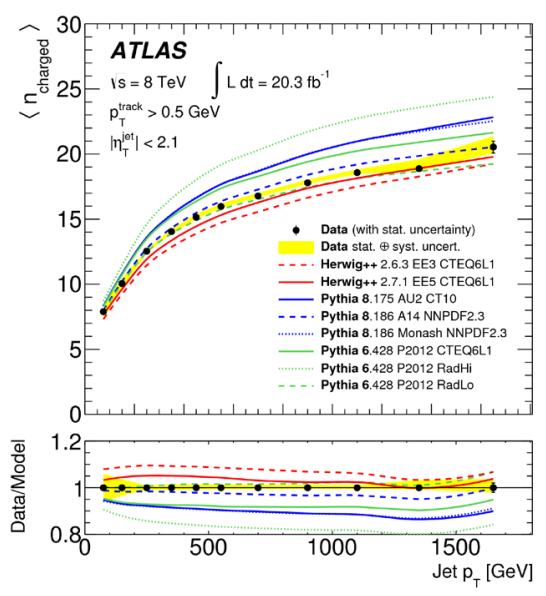

(a)

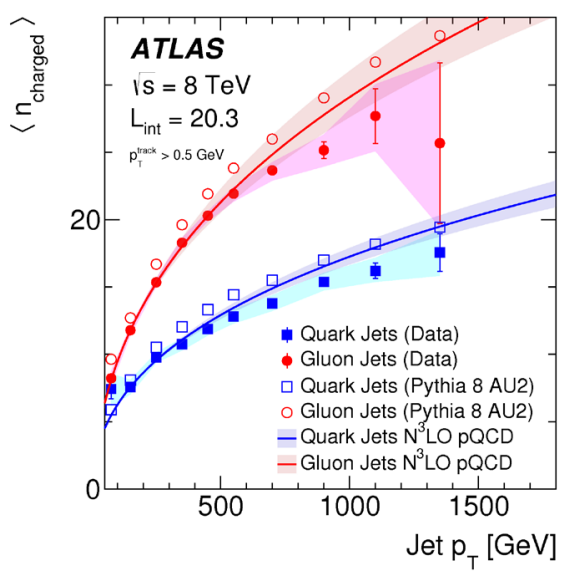

(b)

Figure 4: (a) The measured average charged-particle multiplicity (left) as a function of the jet $\mathrm{p}_{\mathrm{T}}$, combining the more forward and the more central jets for $\mathrm{p}_{\mathrm{T}}{ }^{\text {track }}>0.5 \mathrm{GeV}$. (b)The jet $\mathrm{p}_{\mathrm{T}}$ dependence of the average charged-particle multiplicity $\left(\mathrm{p}_{\mathrm{T}}{ }^{\text {track }}>0.5 \mathrm{GeV}\right)$ for quark- and gluon-initiated jets, extracted with the gluon fractions from PyTHIA 8.175 with the CT10PDF [7].

\section{Inclusive isolated-photon production}

Inclusive isolated-photon production $(p p \rightarrow \gamma+X)$ in $p p$ collisions provides a testing environment for perturbative QCD. It was studied by the ATLAS experiment at $\sqrt{s}=13 \mathrm{TeV}$ [8]. The data sample used for analysis corresponds to an integrated luminosity of $6.4 \mathrm{pb}^{-1}$. A sample of photons with high transverse energies has been selected using the characteristics of the shower shapes in the calorimeter. Both converted and unconverted photons were included in the sample. The photons were required to be isolated by rejecting those candidates in which the transverse energy in a cone of $\Delta \mathrm{R}=0.4^{1}$ around their direction was larger than $4.8 \mathrm{GeV}+4.2 \cdot 10^{-3} \mathrm{E}_{\mathrm{T}}^{\gamma}$. A data-driven subtraction method was applied to remove the background from multi-jet processes, in which a jet is misidentified as a photon. As a result of this analysis, a clear signal of inclusive isolated photons at $\sqrt{s}=13 \mathrm{TeV}$ has been observed for the first time in the phase-space region given by for $\mathrm{E}_{\mathrm{T}}^{\gamma}>125 \mathrm{GeV}$ and $\left|\eta^{\gamma}\right|<2.37$, excluding the region of the ATLAS calorimeter crack between the barrel and the end-caps $(1.37<$ $\left.\left|\eta_{\gamma}\right|<1.56\right)$. The kinematic distributions of the signal yield as functions of $\mathrm{E}^{\gamma} \mathrm{T}$ and $\left|\eta^{\gamma}\right|$ are shown in Figure 5. The measured distributions are compared to the simulated SHERPA events normalised to the total yield observed in the data. The simulation provides a good description of the shape of the measured kinematic distributions.

\footnotetext{
${ }^{1} \Delta \mathrm{R}=\sqrt{(\Delta \eta)^{2}+(\Delta \phi)^{2}}$ defines the distance between objects in $(\eta-\phi)$ space.
} 

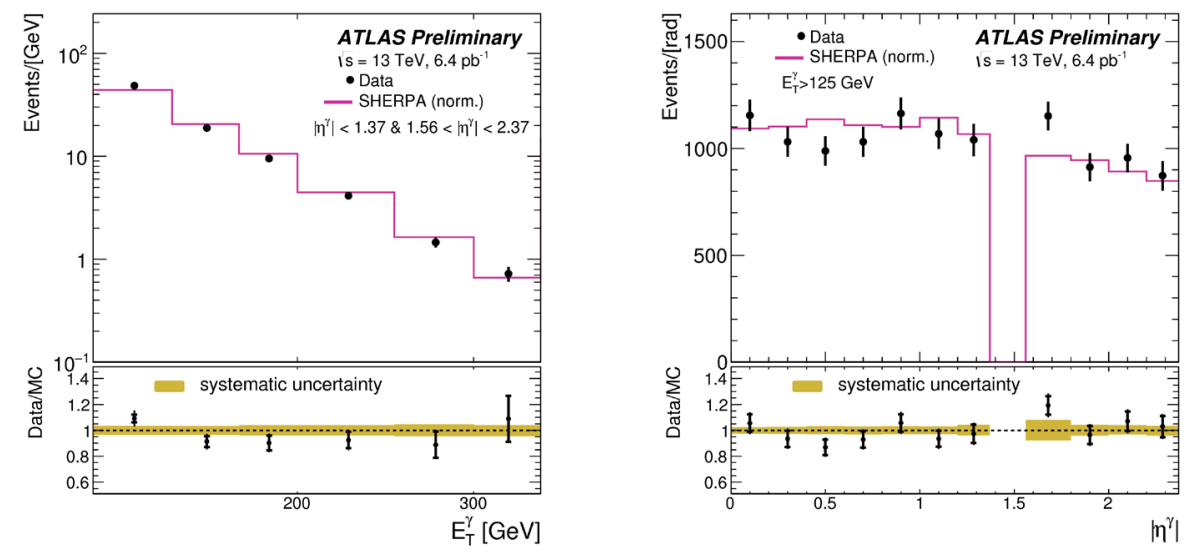

Figure 5: Measured isolated-photon yield (dots) as a function of $\mathrm{E}_{\mathrm{T}}^{\gamma}$ (left) and as a function of $\left|\eta^{\gamma}\right|$ for $\mathrm{E}_{\mathrm{T}}^{\gamma}>125 \mathrm{GeV}$ (right); the error bars represent the statistical uncertainty of the data. The SHERPA MC simulations, normalised to the integrated measured signal yield, (histograms) are also included. The bottom panels of the plots show the ratio of the data and the MC simulation (dots). The inner error bars represent the statistical uncertainty of the data and the outer error bars display the uncertainty due to the background subtraction added in quadrature to the statistical uncertainty. The shaded band represents the uncertainty due to the photon energy scale, energy resolution and identification efficiency as well as the trigger efficiency and the modelling of the isolation transverse energy in the MC added in quadrature [8].

\section{Production cross-sections of a $\mathrm{Z}$ boson in association with jets}

The measurement of the weak boson $(\mathrm{Z})$ production cross-section gives another good benchmark for current understanding of the perturbative QCD and electroweak processes. The weak boson production at LHC $p p$ collisions has a relatively large cross-section and boson decays to leptonic final states are a clean experimental signature. Therefore it can be measured with a high precision. The processes with weak bosons production constitute an important background for Higgs boson and new physics searches.

The production cross-section of $\mathrm{Z}$ boson in association with up to four jets was measured using $p p$ collision data collected at $\sqrt{s}=13 \mathrm{TeV}$ and corresponding to a total integrated luminosity of approximately $85 \mathrm{pb}^{-1}$ [9]. The events with $\mathrm{Z}$ bosons decaying to electron or muon pairs, produced in association with one or more jets in the kinematic range of $\mathrm{p}^{\text {jet }} \mathrm{T}>30 \mathrm{GeV}$ and $\left|\mathrm{y}^{\mathrm{jet}}\right|<2.5$, were analysed. The kinematic cuts used in the analysis are described in detail in [9]. The fiducial production cross-sections for $\mathrm{Z}$ bosons and inclusive cross-section to up to four jet multiplicities were measured with a precision ranging from approximately $10 \%$ to approximately $20 \%$ as presented in Figure 6 . Ratios of cross-sections for successive jet multiplicities were derived and are also shown in Figure 6. The measured fiducial cross-sections for the various jet multiplicities and the ratios of successive jet multiplicities were compared to predictions from MC models - SHERPA and MADGraPH. The predictions show a reasonable agreement with the measured cross-sections and cross-section ratios within the uncertainties. 

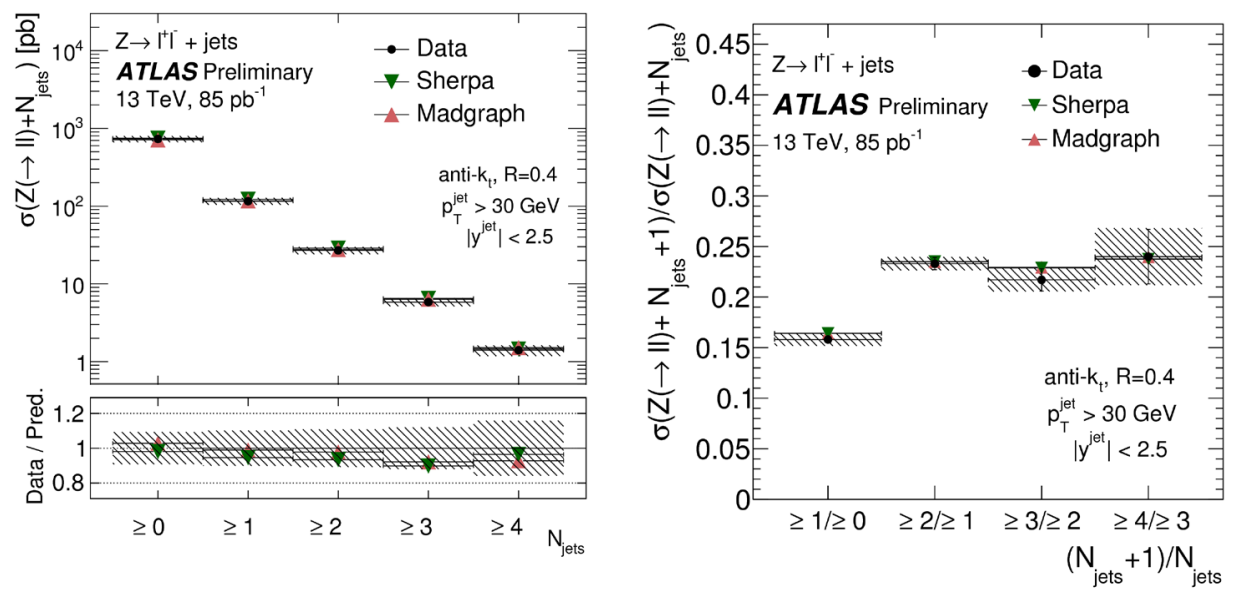

Figure 6: Combined $\mathrm{Z}$ to $l l$ plus $\mathrm{N}$ jets fiducial cross-sections (left), and cross-section ratios for successive jet multiplicities (right). The results are compared to predictions from SHERPA and MADGRAPH. The hatched error band corresponds to the total uncertainty of the results: systematic, statistical, and luminosity uncertainties for the fiducial cross-section results (left), systematic and statistical uncertainties for the ratio results (right) [9].

\section{Summary}

Several recent measurements related to the soft SM processes from the ATLAS experiment are presented for $p p$ collisions at 8 and $13 \mathrm{TeV}$ at the LHC at CERN. Monte Carlo generators, tuned in lower energy regimes, describe the collected data reasonably well. The latest ATLAS public results can be found at https://twiki.cern.ch/twiki/bin/view/AtlasPublic/StandardModelPublicResults.

\section{Acknowledgements}

This work was in part supported in Poland by the National Science Centre grant 2015/19/B/ST2/00906 and by PL-Grid Infrastructure.

\section{References}

[1] ATLAS Collaboration, 2008 JINST 3 S08003.

[2] Evans, L. and Bryant, P., 2008 JINST 3 S08001.

[3] ATLAS Collaboration, https://twiki.cern.ch/twiki/bin/view/AtlasPublic/

LuminosityPublicResultsRun2.

[4] ATLAS Collaboration, ATL-PHYS-PUB-2015-019, http://cds.cern.ch/record/2037684.

[5] ATLAS Collaboration, Phys. Lett. B 758 (2016) 67 [arXiv:1602.01633 [hep-ex]].

[6] ATLAS Collaboration, Eur. Phys. J. C 76 (2016) no.9, 502 [arXiv:1606.01133 [hep-ex]].

[7] ATLAS Collaboration, Eur. Phys. J. C 76, no. 6, 322 (2016) [arXiv:1602.00988 [hep-ex]].

[8] ATLAS collaboration, ATLAS-CONF-2015-034, http://cdsweb.cern.ch/record/2037667.

[9] ATLAS collaboration, ATLAS-CONF-2015-041, http://cdsweb.cern.ch/record/2048104. 